\title{
The Payload Development and the Experiments for Studying Thermocapillary Convection in TG-2 Liquid Bridge
}

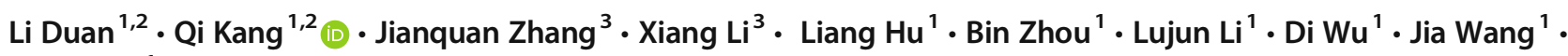 \\ Pu Zhang ${ }^{1}$
}

Received: 13 March 2020 / Accepted: 3 December 2020 / Published online: 23 January 2021

(C) The Author(s) 2021

\begin{abstract}
The development of space experiment payload for studying thermocapillary convection in the liquid bridge with large Pr number on TG-2 space laboratory as well as the experiments are presented in detail in this paper, and the objectives of the space experiments are confirmed. The functions of the payload are analyzed, and the technical and engineering specifications are determined. Detailed designs and experimental verifications are performed on the structure of liquid bridge columns, the method of bubble removing in the liquid, the bridge cleaning system, the accurate control of aspect ratio and volume ratio, and the highsensitivity measurement of fluid temperature. Matching experiments on the ground according to space experiment properties are carried out, $5 \mathrm{cSt}$ silicone oil is selected as the fluid medium in space experiments. And the states of liquid bridge and temperature oscillation signals obtained from space experiments are presented at the end of this paper. Specific summarizations and discussions to the experiment project on fluid science in space are conducted in this paper, which will provide a useful reference for scientists participating space experimental research in the future.
\end{abstract}

Keywords Liquid bridge $\cdot$ Payload development $\cdot$ Experiment verification $\cdot$ Space experiment

\section{Introduction}

Floating zone crystal growth is an important single crystal growth method in materials science. The model of thermocapillary convection in the liquid bridge of half floating zone comes from this kind of material growth system directly. Convection, especially instable convection, is an important factor affecting the quality of crystal growth. In ground experiments, buoyant convection is hardly removable due to gravity, and this kind of convection is usually unsteady. The half floating zone

This article belongs to the Topical Collection: The Effect of Gravity on Physical and Biological Phenomena

Guest Editor: Valentina Shevtsova

Qi Kang

kq@imech.ac.cn

1 Key Laboratory of Microgravity, Institute of Mechanics Chinese Academy of Sciences Beijing 100190, People's Republic of China

2 School of Engineering Sciences University of Chinese Academy of Sciences Beijing 100049, People's Republic of China

3 Technology and Engineering Center for Space Utilization Chinese Academy of Sciences Beijing 100094, People's Republic of China method of crystal growth still has the convection phenomenon because of thermocapillary effect on the surface. Therefore, the study on thermocapillary convection in the liquid bridge has a significant meaning in practical applications.

Today, thermocapillary convection in the liquid bridge of half floating zone has become a typical research project in microgravity fluid physics. Though, studies on thermocapillary convection have been carried out for a long time, and various explanations to the mechanisms of oscillation and transition have been put forward, the instable mechanism of this flow is still an open problem, especially the transition route is very complicated, and further theoretical and experimental studies are required. Since it is difficult to carry out experimental study on large-size liquid bridges on the ground, the experiment ranges of control parameters are limited significantly, in addition, the experiments on small-size liquid bridges make the diagnosis very difficult, as a result, there is a big difference between the experiment results and theoretical analysis results. Space experimental study on this problem has a theoretical significance to understand instability and transition processes of thermocapillary convection in depth.

Floating zone method is an important technology for high quality crystal growth. On the ground, the diameter of growing 
crystal is limited by gravity. With the development of space technology, growing high quality large-size crystal under the microgravity condition in spacecraft becomes possible due to the significant decrease of static pressure and buoyant convection. Therefore, the floating zone method of crystal growth in space is the most promising technology in industry, in addition, it has a guiding effect on crystal growth with floating zone method on the ground. However, with the weakening of gravity, some unimportant factors acting on the fluid on the ground become prominent in space. For example, the non-uniform temperature distribution on free surface of floating zone will cause thermocapillary convection driven by the surface tension gradient. The instability of this convection is an issue that must be taken into account in crystal growth in space.

In order to understand the law of thermocapillary convection, studies on thermocapillary convection in half floating zone (liquid bridge) have been carried out in many countries since 1970s. In the end of 1970s, Chun and Schwabe found almost at the same time in their experiments on thermocapillary convection in the half floating zone liquid bridge that (Schwabe et al. 1978; Chun and Wuest 1979), when the temperature difference between the top and bottom of the half floating zone was greater than a critical value, the convection driven by surface tension gradient would lose stability and start to oscillate. The convection oscillation will cause the temperature oscillation, as a result, the growth striations will appear in the growing crystal, which will affect the crystal quality. Thereafter, with the rapid development of space technology, there are more and more opportunities for space experiments. As a matter of course, understanding the oscillation mechanism of thermocapillary convection and developing the controlling oscillation ways have become the study focus of microgravity fluid science in the world. In the beginning of 1980s, Smith and Davis (1983) found that, due to the effect of temperature gradient on the liquid-air interface of liquid layer, there would be hydrothermal waves propagating in the fluid if the temperature of fluid is higher than a critical value; they believed that the stable thermocapillary convection in the liquid bridge of half floating zone mentioned by Chun and Schwabe had the similar flow structure as the two-dimensional back-flow in the liquid layer; and they deducted that the mechanism of forming hydrothermal waves in an infinite liquid layer would be the same as the mechanism of oscillation instability in the halffloating zone liquid bridge with a free surface. Smith and Davis reckoned that the free surface deformation played a key role in starting the instability, and the coupling of speed and temperature field with free surface deformation led to thermocapillary oscillation.

Ostrach et al. (Kamotani et al. 2000, 1995, 1994, 1998) studied the stability of thermocapillary convection in the liquid bridge and in the cylindrical liquid layer, and they believed that free surface deformation was the key reason for starting thermocapillary instability, and the time lag between surface flow and back flow as well as the response of the velocity field to the temperature field on the surface caused thermocapillary oscillation. Since 2008, JAXA (Kawamura et al. 2012; Nishino et al. 2015) has carried out three Marangoni experiments in space (MEIS) to study thermocapillary convection in liquid bridges on the international space station (ISS). Various experimental methods have been adopted in their experiments, including 3D-PTV, side-looking camera, thermal imager, thermocouple sensors, and photochromic technology. In 2008, MEIS-1 was finished with the experiment medium of $5 \mathrm{cSt}$ silicone oil $(\operatorname{Pr}=67)$, the diameter of bridge column was $30 \mathrm{~mm}$, the maximum liquid bridge height was $60 \mathrm{~mm}$. The critical temperature difference of thermocapillary convection was determined, the values of $\Delta T_{c}$ and $M a_{c}$ of oscillating flow corresponding to different aspect ratios $(0.225 \sim 1.25)$ were given, and the accumulation structure of particles was observed. In 2009, MEIS-2 was finished with the experiment medium of $5 \mathrm{cSt}$ silicone oil $(\mathrm{Pr}=67)$, the diameter of bridge column was $30 \mathrm{~mm}$, the maximum liquid bridge height was $60 \mathrm{~mm}$. The critical temperature differences in a wider range of aspect ratio were determined, and the surface velocity was measured with the photochromic technology. In 2010, MEIS-
Fig. 1 The experimental schematic for studying temperature oscillation in liquid bridge

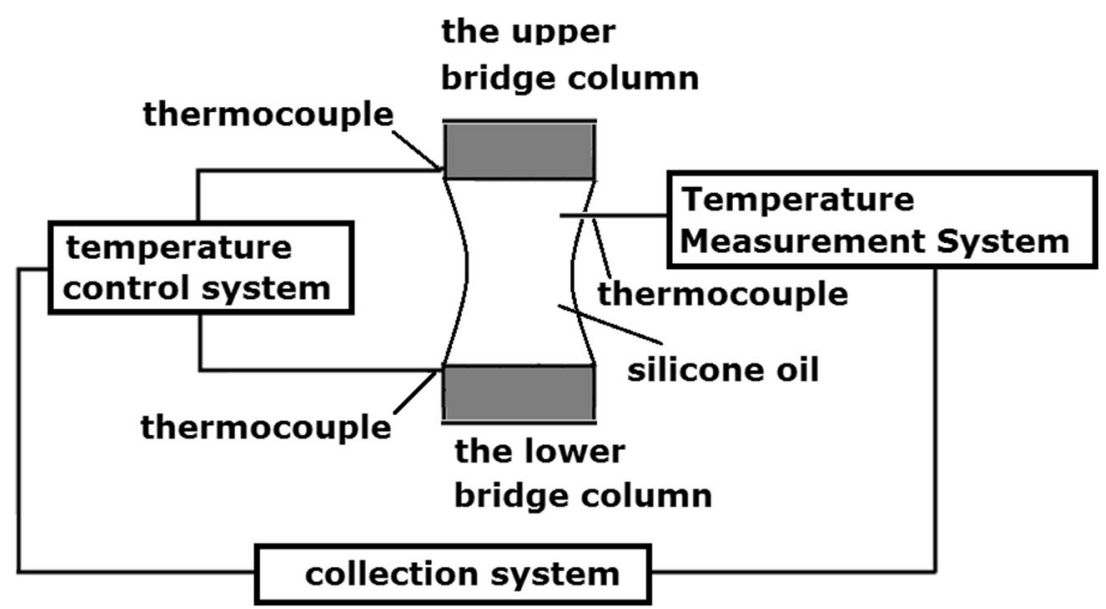


Fig. 2 The design of experiment setup

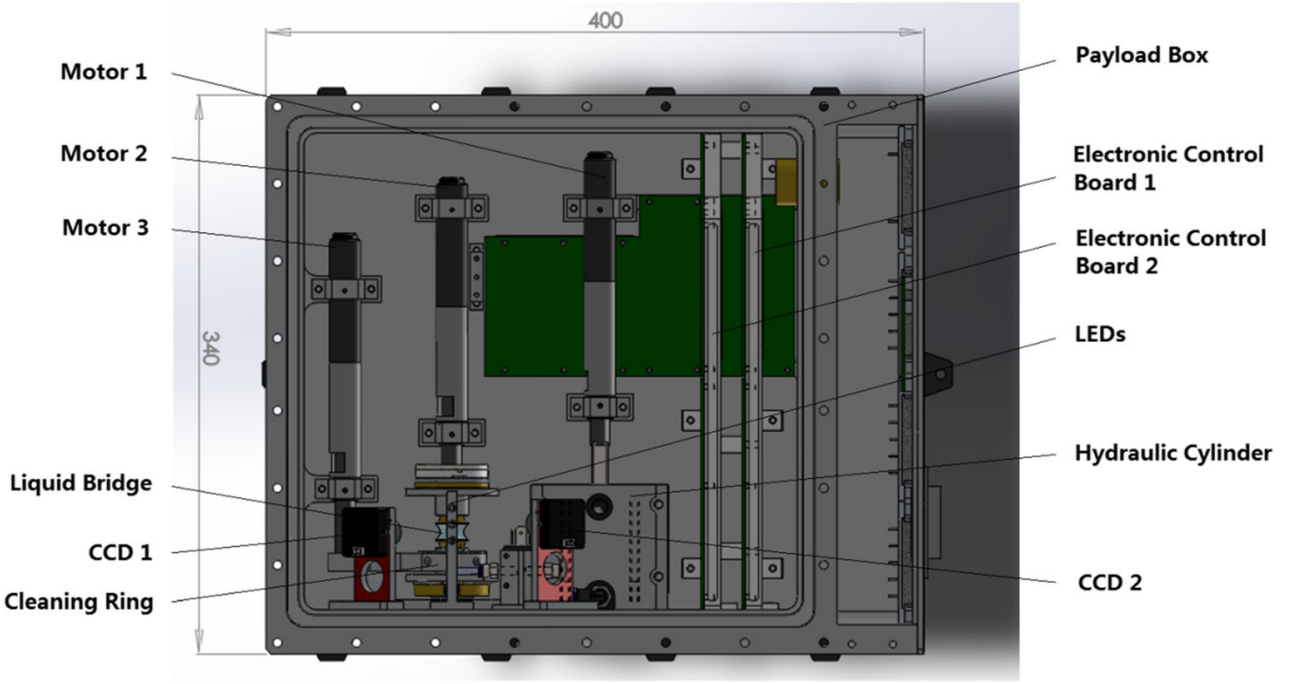

4 was finished with the experiment medium of 20cSt silicone oil $(\operatorname{Pr}=207)$, the diameter of bridge column was $50 \mathrm{~mm}$, the maximum liquid bridge height was $62.5 \mathrm{~mm}$. It was found that the second harmonic frequency would appear in the temperature oscillation, and the hydrothermal wave propagating in the radial direction from the hot end to the cold end was observed; with the increase of Marangoni number, the oscillation mode was the standing wave first, then the rotating wave, and finally in the chaotic state. From the experimental object, experimental model, and experimental methods of Japanese space experimental studies on thermocapillary convection in the liquid bridge, we can see that, Japan has invested significant resources into the space experimental study on thermocapillary convection, including space resources, human resources, material resources, and funding resources, which we cannot compare with.

ESA has always attached great importance to the microgravity fluid research. Shevtsova et al. (2014) reported the ESA/JAXA experiment called JEREMI planned on the ISS in 2014. The physical problem that the cylindrical liquid bridge is concentrically surrounded by an annular gas channel has been considered and studied. A few three-dimensional numerical codes for one- and two-phase simulations and different experimental setups have been developed. The numerical and experimental analyses have proved that dynamic freesurface deformation can be neglected in numerical modes if the Reynolds number in the gas phase satisfies $\left|R e^{g}\right| \leq 100$.

Since the end of 1980s, detailed theoretical studies, numerical simulations and ground experiments have been carried out on the convection in the liquid bridge of half floating zone in our Country (Hu et al. 2009, 1994; Hu and Tang 2013). The physical model and mathematical model of thermocapillary convection in the liquid bridge have been established, and the basic characteristics of this convection have been given. Various experimental methods have been adopted in the study of mechanism of convection, convection oscillation, critical condition and transition process in the small-size liquid bridge. Basic characteristics of thermocapillary convection in the liquid bridge at different stages were given, which had direct relations with the aspect ratio and volume ratio of the
Fig. 3 The space experiment payload

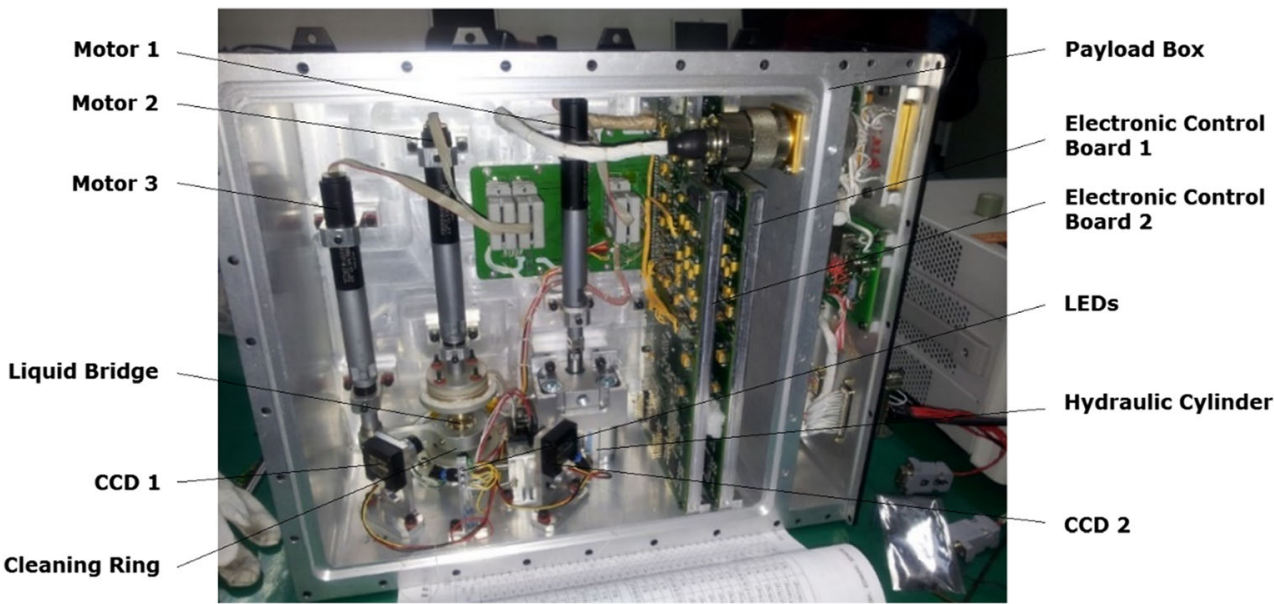


liquid bridge. The fluctuating deformation on the free surface was observed, and two transitions in the liquid bridge were found. Both numerical and experimental studies have obtained the subharmonic bifurcation process from laminar flow to turbulent flow of thermocapillary convection. In recent years, Kang et al. (2019, 2019, 2019; Zhang 2014) carried out space experimental studies on annular thermocapillary convection on SJ-10 recoverable satellite of China, and obtained the oscillation characteristics, critical conditions, transition processes, as well as transformations between the standing wave and the travelling wave of thermocapillary convection in space.

This paper introduces the development of payload for the space experimental project on thermocapillary convection in the liquid bridge carried out in the space laboratory as well as the corresponding ground and space experimental results. The objective of this space experiment is to study the influences of aspect ratio, $A r=L / D$, and volume ratio, $V r=V / V_{0}$, on the critical process of thermocapillary convection in the liquid bridge, and to discuss the second transition, and so on.

\section{Research Methods and Experimental Plan}

As a fluid mechanics system, thermocapillary convection interests many researchers in its oscillation characteristics and the whole transition process from steady flow to turbulent flow. The experimental method is shown in Fig. 1. The upper bridge column has a heater for heating the top of the liquid bridge, and the heating rate is controllable. The lower bridge column has a temperature controller to maintain the bottom of the liquid bridge at a low temperature. The temperature on the top column is $T=T_{0}+\Delta T$, and the temperature on the bottom column is $T_{0}$. The un-uniform of temperature on the free surface leads to the un-uniform of surface tension, which drives thermocapillary convection. During the experimental process, thermocouples are used to measure temperatures on the top and bottom columns and the temperature inside the liquid bridge. With the increase of applied temperature difference, $\Delta T$, the temperature inside the liquid bridge increases too. The dimensionless parameter of critical Marangoni number corresponding to the critical applied temperature difference, $\Delta T_{c}$, is defined as:

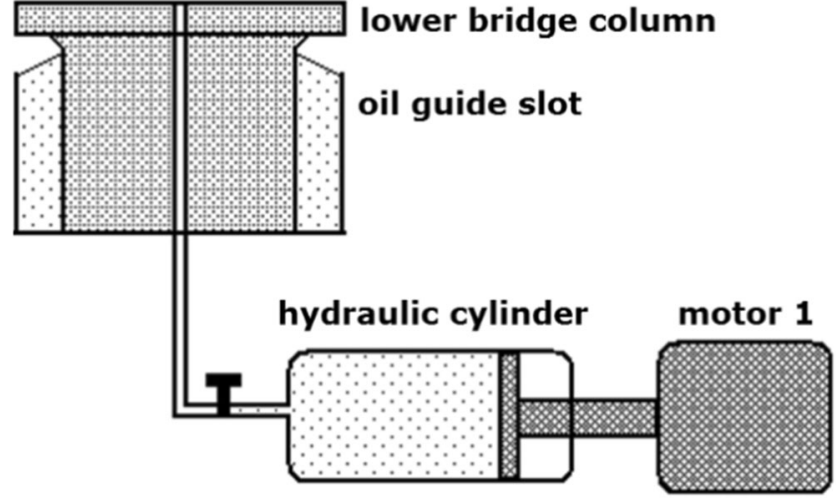

Fig. 5 The liquid store and filling system

$M a_{c}=\frac{\left(\sigma_{T}^{\prime}\right) \cdot\left(\Delta T_{c}\right) \cdot l}{\rho \kappa \mu}$

The ground experiments have proved that two transitions occur before the convection oscillation starts (Wang et al. 2017; Wenrui et al. 1993; Zemei et al. 2002); the first transition is from the steady axisymmetric field to steady nonaxisymmetric field, and at this time, the temperatures of points on the same circumference of the same cross section in the liquid bridge change from equal to different; when $\Delta T$ exceeds a critical value $\Delta T_{c}$, that is, when the Marangoni number exceeds a critical value, the second transition occurs from steady non-axisymmetric field to oscillation state, and the temperature inside the liquid bridge starts to oscillate.

After the oscillation starts, in the transition process to chaos, some phenomena such as quasi-period and perioddoubling bifurcation will occur in thermocapillary convection (Zhu et al. 2011, 2013; Jiang et al. 2017a, b). In the space experiments, the temperatures measured by 5 thermocouples are used to discuss transition processes and wave relations.

The process of transition and bifurcation, as well as the second transition in thermocapillary convection in the liquid bridge can be obtained by measuring and analyzing temperature evolution of a point with time; by comparing oscillation phases of various temperature points inside the liquid bridge, the hydrothermal wave problem can be analyzed. By changing the height of liquid bridge, $L$, the relationship between the critical Marangoni number and the aspect ratio, $A r=L / D$ can be analyzed; by changing the liquid volume of the liquid bridge, $V$, the influence of volume ratio of the liquid bridge,
Fig. 4 The overall structure diagrams of liquid bridge
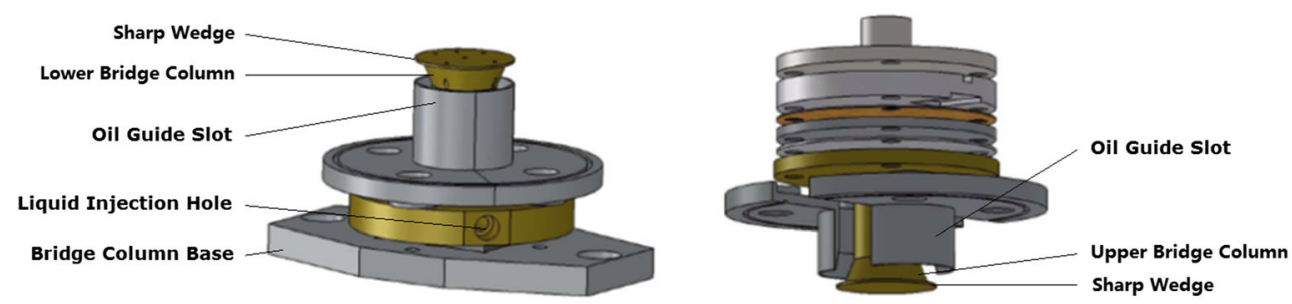


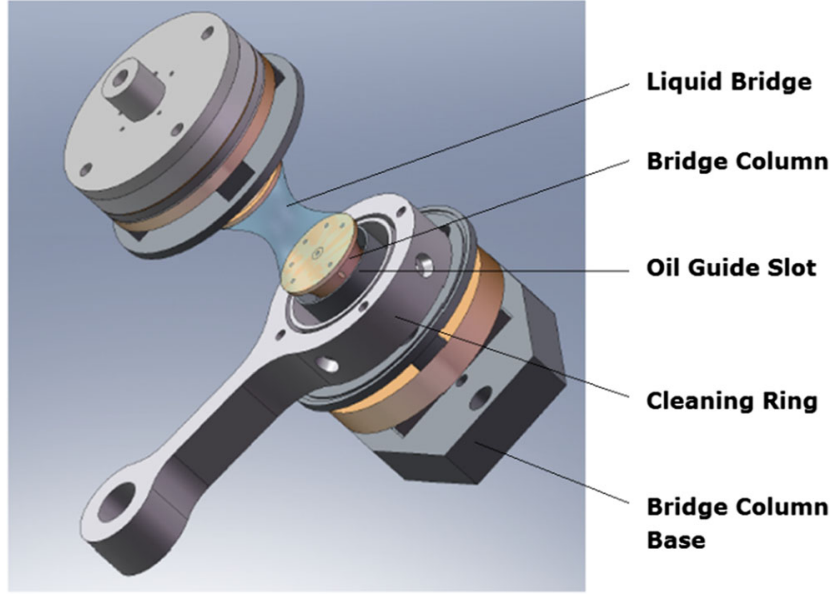

Fig. 6 The working principle of bridge cleaning

$V / V_{0}$ ), (where, $V_{0}$ is the column volume of the liquid bridge) on the critical oscillation can be studied. Usually, a slender bridge ( $V / V_{0}$ is smaller than the demarcation value) and a fat bridge ( $V / V_{0}$ is larger than the demarcation value) have different starting oscillation laws and different oscillation modes. The volume effect of liquid bridge with a large Prandtl number can be studied through comprehensive analysis of the fluid temperature changes.

The objectives of our space experiments are to study influences of aspect ratio and volume ratio of liquid bridge on the critical process of thermocapillary convection, and to discuss the second transition. In view of the above research objectives, in the development of space experimental payload, five thermocouples are laid out on the same cross section in the liquid bridge to measure the temperature of five points under different aspect ratios and volume ratios of the liquid bridge, to finish the scientific objectives of this project. The volume ratio of the liquid bridge is calculated by adding liquid accurately with the liquid filling motor, and the aspect ratio of the liquid bridge is determined by pulling the bridge precisely with the bridge pulling motor. Two CCD cameras as engineering backups are used to monitor the liquid bridge state in distant view and close shot respectively. The image captured by distant view shows the complete state of liquid bridge, and the image captured by close shot shows the state in the bridge corner, as shown in Fig. 17. Besides, the images are also used for judging whether the liquid bridge has been established, and verifying the aspect ratio and volume ratio of the liquid bridge.

Microgravity level is the main factor that affects the results of space experiments. In order to minimize the influence of $\mathrm{g}$ Jitter in the TG-2 space laboratory, the microgravity level is required to be lower than $10^{-3} \mathrm{~g}$ during space experiments. The temperature of working environment is in the range of $10^{\circ} \mathrm{C} \sim 35^{\circ} \mathrm{C}$, and there is no humidity requirement. This payload is a sealed chamber, so there is no requirement in pressure, radiation and illumination.

\section{Design and Development of The Payload}

The space experimental payload of the liquid bridge is made up of the liquid bridge and pulling system, liquid store and filling system, bridge cleaning system, two-way temperature control system, multi-channel temperature measuring system, light source system, image acquisition system, and electronic control system. The experimental setup is designed as shown in Fig. 2. In the payload box with a volume of $340 \mathrm{~mm} \cdot 150 \mathrm{~mm} \cdot 400 \mathrm{~mm}$, the left hand side is a sealed chamber, and inside are the liquid bridge and pulling system (motor 2), liquid store (hydraulic cylinder) and filling system (motor 1), bridge clearing system (cleaning ring), temperature controller, temperature data collector, image acquisition system (CCD 1 and CCD 2), and electronic control system; the right hand side is not sealed, and inside are the DCDC power supply and all the sockets.
Fig. 7 Installation of 5 thermocouples
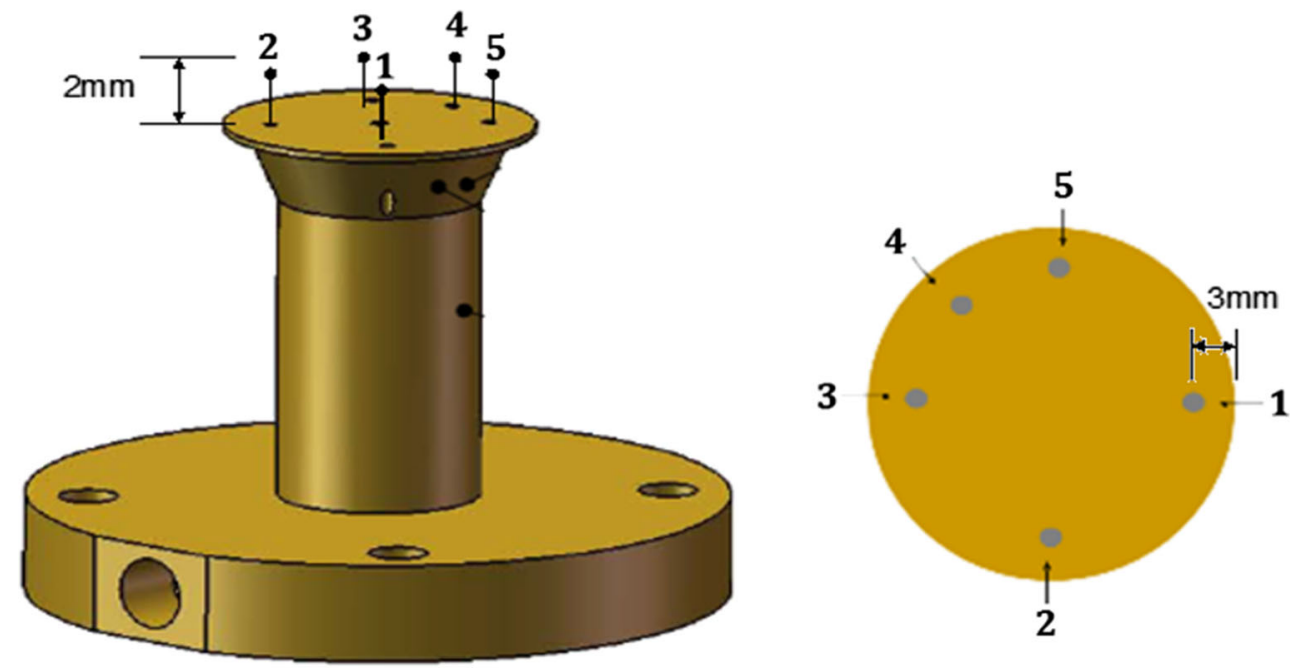
Fig. 8 The LED illumination system and image acquisition system

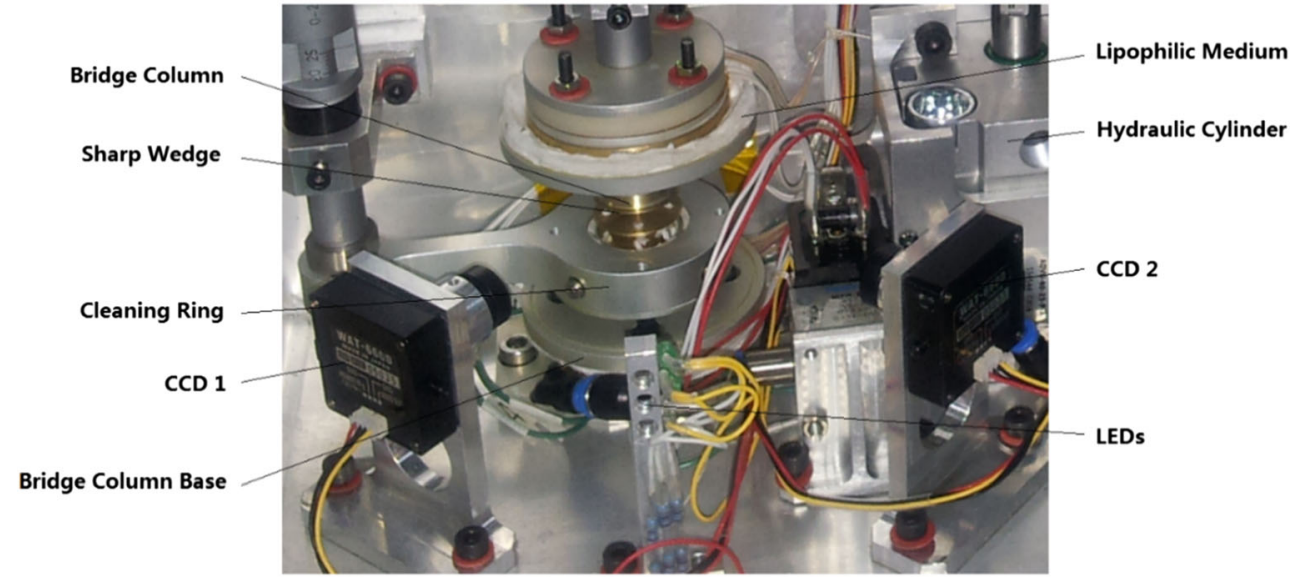

The space experimental payload is shown in Fig. 3. The experimental sample of silicone oil is stored in the hydraulic cylinder before the start of space experiments. With the coordination work of liquid filling motor (motor 1) and the bridge pulling motor (motor 2), the liquid filling rate and the bridge pulling rate are controlled to establish the liquid bridge with different aspect ratios and volume ratios. The LED light source is used to illuminate the liquid bridge, and the CCD image acquisition system is used to record the state of liquid bridge during the experimental process. The upper bridge column is heated with an electro thermal film, and the lower bridge column is cooled with Peltier element. Thermocapillary convection is formed in the liquid bridge due to the temperature difference between the upper and lower bridge columns, and the temperature difference is controlled by the temperature control system. Thermocouples are used to measure temperatures of 5 points on the same cross section in the liquid bridge. If the bridge breaking happens in space experiments, the bridge clearing system is started to clear the bridge columns and the liquid bridge will be established again. The control systems in the experimental setup are in charge of the coordination work among all the systems to accomplish space experiments. The whole space experiments consist of independent single experiments, and the duration for each single experiment is $90 \sim 120 \mathrm{~min}$. For different aspect ratios, volume ratios, and heating modes, there could be hundreds of different experimental conditions, and 1-5 experiments can be carried out every day.

The liquid bridge and the bridge pulling system consists of the upper bridge column, the lower bridge column and the motor 2, as shown in Figs. 2 and 3. The diagrams of bridge columns are shown in Fig. 4, where, the left is the lower bridge column and the right is the upper bridge column, and the diameter of bridge columns is $20 \mathrm{~mm}$. It is an important feature under microgravity that liquid crawls along the solid surface. In space experiments, in order to establish the liquid bridge, effective measures should be taken to prevent the liquid from crawling along bridge columns. Two methods have been designed, one is adopting sharp wedges on the surface edges of bridge columns, and the other is applying anti-climbing liquid below the sharp wedges. These two methods have a good effect to prevent the experiment medium, silicone oil, from crawling along solid surfaces. There are oil guide slots on the bridge columns. The lipophilic medium is filled in the oil guide slots extending to the bases of bridge columns. The base of the lower bridge column is connected to a wall of the payload box, and the wall is also covered with lipophilic medium. Once the liquid bridge is broken in space experiments, the silicone oil will flow along the lipophilic medium in the oil guide slots to the bases of bridge columns as well as to the wall of the payload box. Therefore, a large amount of silicone oil will be collected in the lipophilic medium, and a little of
Fig. 9 Working mode Conversions of liquid bridge control software

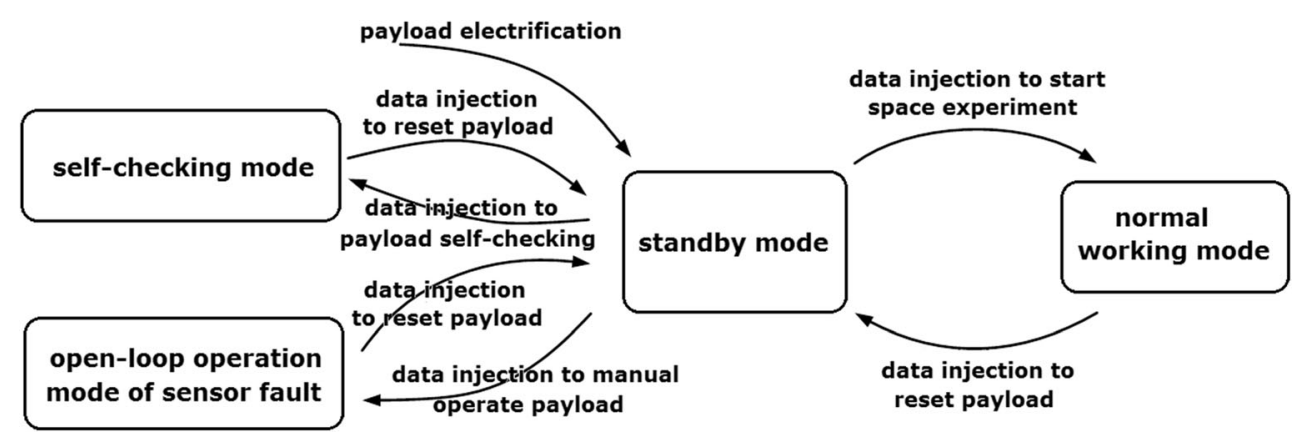


Fig. 10 The bridge cleaning process
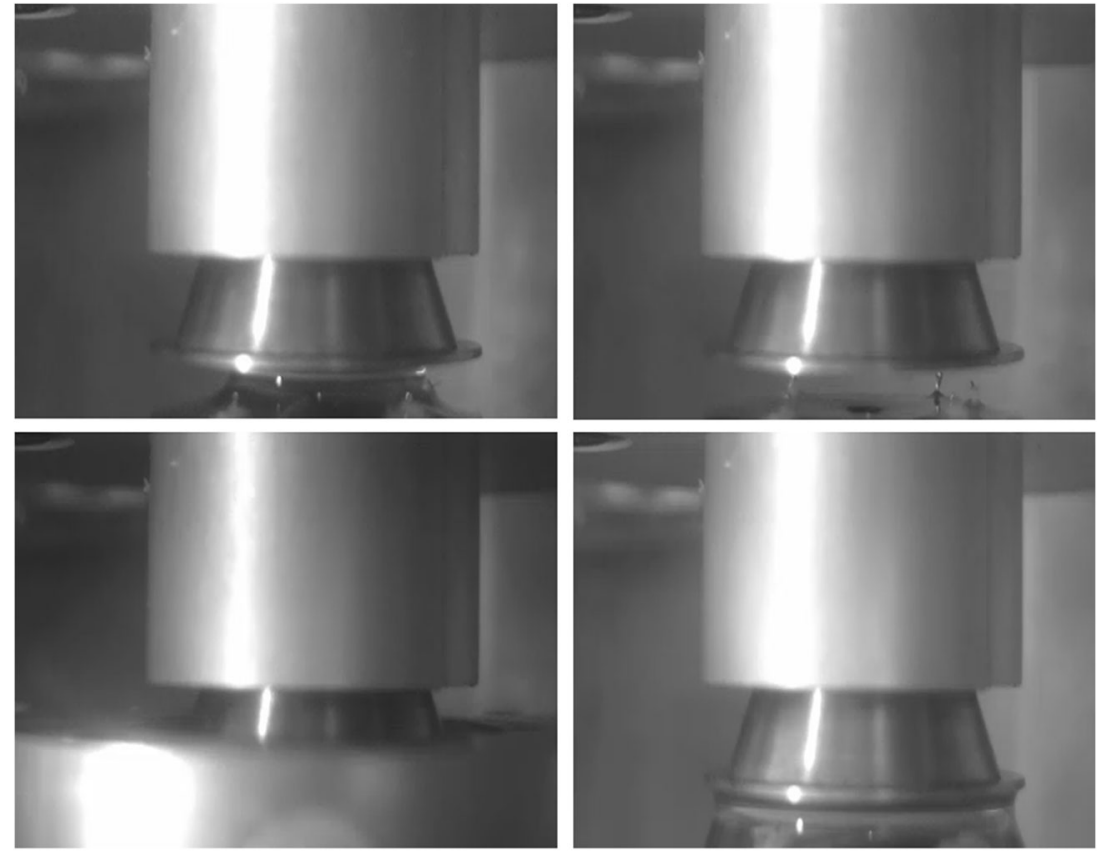

silicone oil left on the bridge columns will be wiped clean by the bridge cleaner. In the experiments, the position of the lower bridge column is fixed, and the liquid bridge is established by using motor 2 to control the position of the upper bridge column. The bridge pulling motor (motor 2) and the liquid filling motor (motor 1) are controlled by DSP, and the control steps are decided by the predetermined conditions of experiments. DSP controls the solenoid valve and opens the liquid filling channel, and then starts the liquid filling motor; in the meantime, DSP controls the bridge pulling motor to move up slowly, forming the liquid bridge gradually. Different aspect ratios of the liquid bridge, $H / D$, can be realized by controlling the pulled displacement. The bridge columns are made of copper, and the liquid filling hole is in the center of the lower bridge column. On the lower bridge column, there are 5 small holes for arranging and inserting thermocouples. The upper bridge column is heated with an electrothermal film to be the hot end of liquid bridge, and the lower bridge column is cooled with Peltier element to be the cold end of liquid bridge, and at this time, the system of thermocapillary convection in the liquid bridge has been formed. During the process of establishing the liquid bridge, slow and stable operation and keeping the upper and lower bridge columns coaxial are the key factors to guarantee the success of experiments, therefore, the upper bridge column is connected to the high precision stepping motor directly.

The liquid store and filling system consists of the hydraulic cylinder, motor 1 and solenoid valve, as shown in Fig. 5. The experiment medium is stored in the hydraulic cylinder before the beginning of experiments; in the experiments, stepping motor 1 pushes the piston of cylinder to fill the experimental fluid medium, silicone oil, into the space between the upper and lower bridge columns to form the liquid bridge. Different volume ratios of the liquid bridge, $V / V_{0}$, can be realized by controlling the liquid filling amount.

The liquid bridge is established by adding the experimental liquid, silicone oil, into the space between the upper and lower bridge columns to form a liquid column, and the surface of liquid column is the liquid free surface, which is kept by the surface tension of liquid. During the experimental process, all kinds of factors can lead to the bridge breaking to happen. In the normal ground experiments, the bridge columns are always cleaned with alcohol. However, due to the limitation of space experimental condition, a proper bridge cleaning
Fig. 11 Results of bubble escaping and dissolution. $\mathbf{a} \boldsymbol{t}=$ $30 \mathrm{~min}$. b $\boldsymbol{t}=60 \mathrm{~min}$. $\mathbf{c} \boldsymbol{t}=90 \mathrm{~min}$

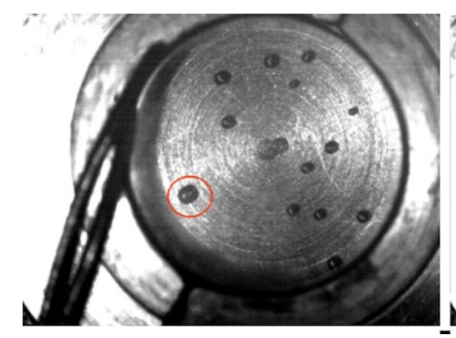

a

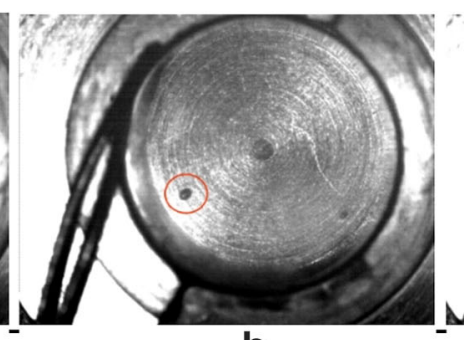

b

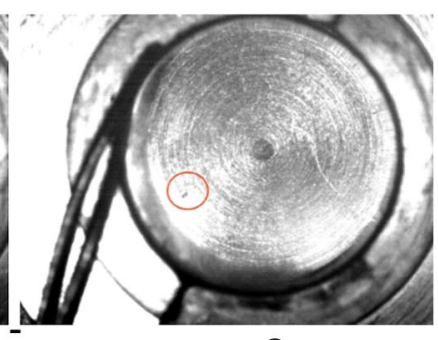

C 


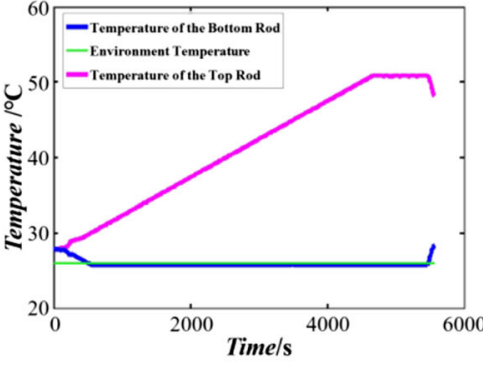

a

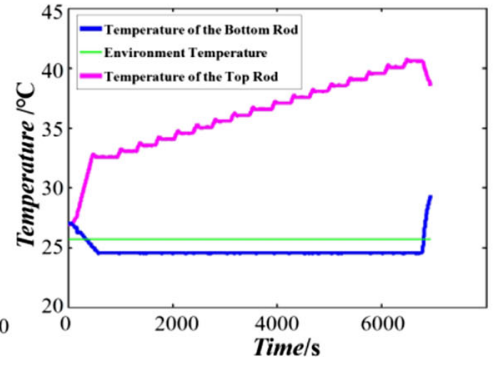

b

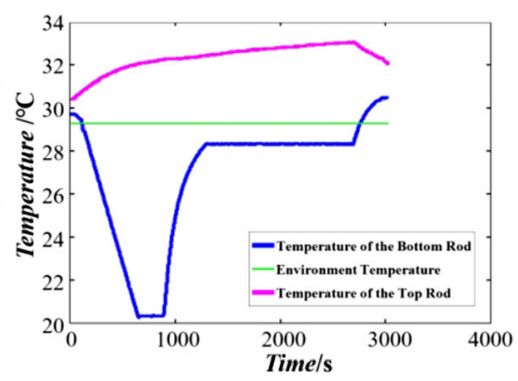

C

Fig. 12 Three typical temperature control modes. a linear heating mode. $\mathbf{b}$ step heating mode. $\mathbf{c}$ bubble removing mode

system need to design. The bridge cleaning system consists of the cleaning ring, lipophilic medium, oil guide slots, and motor 3 , and the working principle of bridge cleaning is shown in Fig. 6. There are oil guide slots on the bridge columns, and the lipophilic medium is filled in the oil guide slots. Once the bridge breaking happens in space experiments, the silicone oil will flow into the oil guide slots along the bridge column, and a little leftover of silicone oil around the bridge column will be wiped clean with the cleaning ring that is driven by motor 3. This method solved the problem of bridge cleaning very well in space experiments.

In space experiments, there are 8 thermocouples in the thermocapillary convection system. Two of them measure the temperature of the cold side and the hot side respectively as feedback signals for PID closed-loop control. The other 6 thermocouples are used to measure the internal temperature oscillations of the fluid.

The two-way temperature control system is made up of the electrothermal film, Peltier element, temperature controller and thermocouples. The heating mode and heating rate can be set by the controller. To control temperatures on the upper and lower columns of the liquid bridge, the upper bridge column is heated with an electrothermal film, and the lower bridge column is cooled with Peltier element, to establish the temperature difference required between the upper and lower ends of the liquid bridge. The temperature control system uses PID closed-loop control algorithm to realize the real time acquisition and control of temperature. The stability of temperature control can reach $\pm 0.5^{\circ} \mathrm{C}$.

The multi-channel temperature measuring system is made up of 6 thermocouples and the temperature collector. It is

Table 1 The parameters of silicone oil

\begin{tabular}{lll}
\hline Silicon oil & Viscosity coefficient & Surface tension coefficient \\
\hline KF96- & $\gamma\left(25^{\circ}\right) \cdot 10^{-6} \mathrm{~m}^{2} / \mathrm{s}$ & $\sigma\left(25^{\circ}\right) \cdot 10^{-3} \mathrm{~N} / \mathrm{m}$ \\
KF96-2 & 2 & 18.3 \\
KF96-5 & 5 & 19.7 \\
KF96-10 & 10 & 20.1 \\
\hline
\end{tabular}

designed as an open-loop mode, that is, the input signal will not be affected by the output signal. So, the measurement results of thermocouples will not affect the temperature control system during experiments. Multi-channel unpackaged thermocouples are used to measure temperatures inside the fluid of liquid bridge. 5 thermocouples are inserted into the fluid through 5 small holes on the lower bridge column and arranged at 5 locations with different azimuth angles $\left(0^{\circ}, 90^{\circ}\right.$, $180^{\circ}, 225^{\circ}$ and $270^{\circ}$ ) on the same cross section, shown as in Fig. 7. And the environment temperature is measured by the sixth thermocouple. The electrical signals of thermocouples are amplified and converted into temperature values, which are sent to the controller for data collecting and storing. The sampling rate is $20 \mathrm{~Hz}$, the consistency of measurement is better than $0.5 \%$, and the sensitivity of temperature measurement is $\pm 0.03^{\circ} \mathrm{C}$. In space experiments, the establishment of the temperature difference between the upper and lower bridges is the most critical. In order to ensure the reliability of the experiment, PID controls are applied by using two thermocouple signals as feedbacks to control the temperature difference between the upper and lower bridge columns. The sampling rate of these two thermocouples is $2 \mathrm{~Hz}$, the consistency is better than $0.5 \%$, and the stability of temperature control is \pm $0.5^{\circ} \mathrm{C}$.

The LED illumination system and the image acquisition system are shown in Fig. 8. The 5-mm Round White Series LEDs (C513A) produced by CREE, Inc. of USA are used for illumination. They are made with an advanced optical-grade epoxy offering superior high-temperature and high moistureresistance performance, and they provide extremely stable light output over long periods of time. 3 LEDs are in parallel connection for the redundant backup, cooperating with the image system. The power supply of LED is $5 \mathrm{~V}$, and the power is about $1.1 \mathrm{~W}$. The image acquisition system consists of two CCD cameras and the image recorder for videos of close shot and distant view respectively, and the video format is D1(720 × 576)@25fps. The video image per frame and each JPEG picture are bound to corresponding timestamp information, which are used to decide the appearance of liquid bridge and verify various experimental parameters. After digital compression by the image card, video signals are sent to the 
Table 2 Matching experiments with 2cSt silicone oil

\begin{tabular}{lllllllllrr}
\hline No. & $\begin{array}{l}H \\
\mathrm{~mm}\end{array}$ & $\begin{array}{l}A r \\
L / D\end{array}$ & $\begin{array}{l}V r \\
V / V_{0}\end{array}$ & $\begin{array}{l}P D \\
\mathrm{~mm}\end{array}$ & $\begin{array}{l}V_{0} \\
\mathrm{~cm}^{3}\end{array}$ & $\begin{array}{l}V \\
\mathrm{~cm}^{3}\end{array}$ & \multicolumn{1}{l}{$\begin{array}{l}L V \\
\mathrm{~mL}\end{array}$} & $\begin{array}{l}P S \\
\mathrm{~mm}\end{array}$ & $\begin{array}{l}\Delta T_{c} \\
{ }^{\circ} \mathrm{C}\end{array}$ \\
\hline 1 & 3.0 & 0.15 & 0.80 & 0 & 0.9420 & 0.7536 & 0.7536 & 0.6000 & 15.07 & 25,380 \\
2 & 3.0 & 0.15 & 0.90 & 0 & 0.9420 & 0.8478 & 0.0942 & 0.0750 & 15.68 & 26,409 \\
3 & 3.5 & 0.175 & 0.80 & 0.5 & 1.0990 & 0.8792 & 0.0314 & 0.0250 & 9.44 & 18,548 \\
4 & 3.5 & 0.175 & 0.90 & 0 & 1.0990 & 0.9891 & 0.1099 & 0.0875 & 10.17 & 19,978 \\
5 & 4.0 & 0.20 & 0.80 & 0.5 & 1.2560 & 1.0048 & 0.0157 & 0.0125 & 8.78 & 19,708 \\
6 & 4.0 & 0.20 & 0.90 & 0 & 1.2560 & 1.1304 & 0.1256 & 0.1000 & 9.61 & 21,577 \\
7 & 4.0 & 0.20 & 0.70 & 0 & 1.2560 & 0.8792 & -0.2512 & -0.2000 & 10.96 & 24,616 \\
8 & 3.5 & 0.175 & 0.70 & -0.5 & 1.0990 & 0.7693 & -0.1099 & -0.0875 & 19.27 & 37,863 \\
9 & 3.0 & 0.15 & 0.70 & -0.5 & 0.9420 & 0.6594 & -0.1099 & -0.0875 & 16.19 & 31,807 \\
\hline
\end{tabular}

controller (data size: 2Mbps). The images provide great convenience for the experiments, as we can see the actual conditions of the liquid bridge clearly. The image system obtains video streams with CCD cameras, and outputs standard PAL/ NTSC video signals. The video streams output from CCD cameras are compressed by the video compression unit and then transferred in packets, supporting $8 \mathrm{~B} 10 \mathrm{~B}$ encoded video stream output with the video data rate less than $3 \mathrm{Mbps}$.

The electronic control system provides conversion functions for power supply from $29 \mathrm{~V}$ to $5 \mathrm{~V}$ and from $29 \mathrm{~V}$ to $12 \mathrm{~V}$ respectively; it conducts motor control, temperature control, temperature signal collection, video signal acquisition, and so on; the collected and package processed data is sent to the scientific experiment management unit through serial main line for down transmission; the electronic control system parses and executes commands of data injection, judges experiment progress of the liquid bridge, and stops the experiment automatically to finish the controlling of the whole experiment process. The main control software of the liquid bridge payload is cured into the internal FLASH of SMJ320F2812, which is real time embedded software. The software system of the liquid bridge payload has 4 working modes when operating in orbit: normal working mode, standby mode, open-loop operating mode in sensor fault, and selfinspection mode. The working mode will be selected by data injection. The conversion relationships between working modes are shown in Fig. 9.

Scientific technical specifications of space experiments:

1) Experiment medium: $5 \mathrm{cSt}$ silicone oil.

2) Diameter of liquid bridge: $20 \mathrm{~mm}$.

3) Aspect ratio: $0.20 \sim 1.10$ variable.

4) Volume ratio: 0.50 1.10 variable.

5) Heating rate: adjustable.

6) Sensitivity of temperature measurement: $\pm 0.03^{\circ} \mathrm{C}$.

7) Maximum temperature difference: $55.00^{\circ} \mathrm{C}$.

8) Stability of temperature control: $0.50^{\circ} \mathrm{C}$.

9) Video image: 2 CCDs.

Engineering technical specifications of space experiments:

1) Average power: $42 \mathrm{~W}$ Operating time: $90 \sim 120 \mathrm{~min} /$ operation.

2) Peak power: $60 \mathrm{~W}$ Operating time: $5 \sim 10 \mathrm{~min} /$ operation.

3) External dimension of experiment box: $340 \mathrm{~mm} \times$ $150 \mathrm{~mm} \times 400 \mathrm{~mm}$.

4) Weight: $13 \pm 0.2 \mathrm{~kg}$.

5) Experimental environment: $10^{\circ} \mathrm{C} \sim 35^{\circ} \mathrm{C}$.

6) The amount of valid data for down transmission in a single experiment: $3.8 \mathrm{G}$ Bytes.

7) Real time transmission rate of valid data to the scientific experiment management unit: $4 \mathrm{M}$ bps.
Fig. 13 Temperature oscillation signals with $2 \mathrm{cSt}$ silicone oil (Heating rate $\mathrm{R}_{\mathrm{tem}}=0.3 / \mathrm{min}$, $\mathrm{T}_{\text {start }}=0 \mathrm{~min}$ )
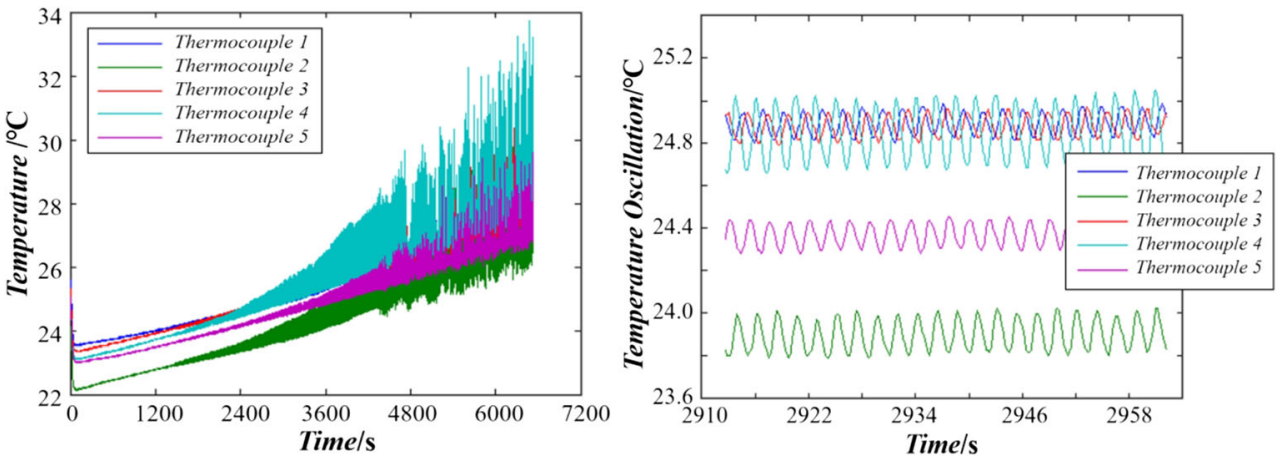
Fig. 14 Tangent bifurcations with $1 / 3 \mathrm{f}$ and $2 / 3 \mathrm{f}$ (Heating rate $\mathrm{R}_{\text {tem }}=0.3 / \mathrm{min}, \mathrm{T}_{\text {start }}=0 \mathrm{~min}$ )
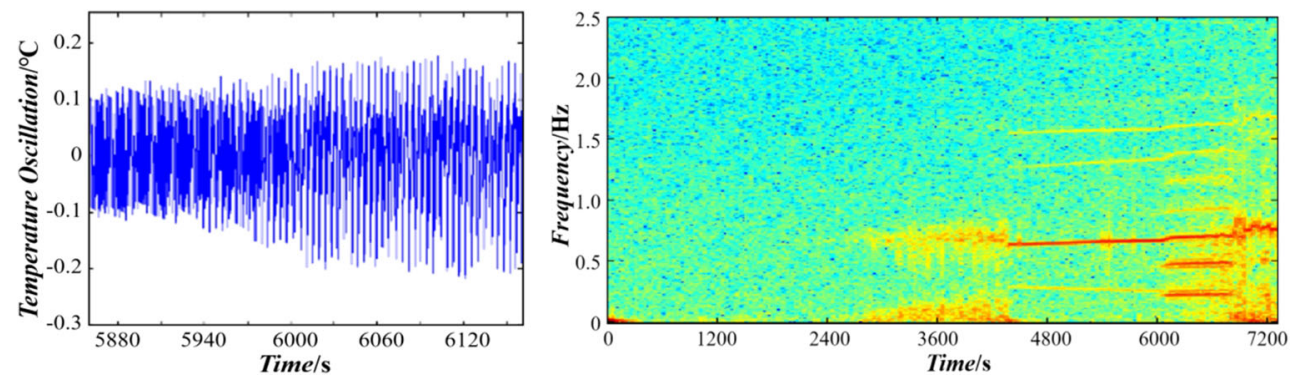

8) External data interface: RS422.

9) Analog remote measurement: 2 lines.

10) Digital remote measurement: 8 bytes.

11) Temperature monitoring point: 1 line.

\section{Experimental Verification on Payload Functions}

\section{Experiment of Bridge Cleaning and Reestablishing}

The experimental verification of bridge cleaning function is shown in Fig. 10. In space experiments, the CCD needs to capture pictures of large liquid bridge, however, only short liquid bridge can be established on the ground, so the liquid bridge in Fig. 10 is at the bottom of pictures. Motor 1 pulls the piston of hydraulic cylinder to suck liquid, which makes the established bridge broken; then motor 3 drives the bridge cleaner to wipe the bridge columns and remove residual liquid, ensuring the wedge of bridge column clean and effectively preventing liquid climbing during the reestablishment of liquid bridge; at last, motor 1 pushes the piston of hydraulic cylinder to fill liquid for reestablishing the liquid bridge. The reestablishing process of liquid bridge is observed. The result shows that the experiment of bridge cleaning and reestablishing is successful.

\section{Experiment of Bubble Removing}

The gas content in the silicone oil of experiment medium is very high, and it is possible that bubbles appear in the liquid bridge during the process of establishing the liquid bridge in space experiments. Once bubbles appear in the liquid bridge, the experimental operation of removing bubbles must be started. The bubbles in the liquid bridge are removed by applying temperature oscillation. As the mechanism of removing bubbles through temperature oscillation is complicated, there is not any related engineering experience that we can learn from. In the ground experiments, the transparent sapphire is used as the upper bridge column, and the bubble removing process can be observed through the bridge column. A series of bubble removing experiments have been performed according to bubble removing modes. By generalizing experiment experience, we found that there are two effective methods of bubble removing: (1) bubble dissolution in the liquid bridge; (2) bubble escaping from the free surface of liquid bridge. Bubble dissolution occurs in the temperature falling stage; bubble escaping occurs mostly in the temperature rising stage, and the higher the heating rate, as well as the greater the temperature difference, the easier the bubble escaping. Since the two methods of bubble removing — bubble escaping and bubble dissolution — are operated in the temperature falling stage and temperature rising stage respectively, the process of bubble removing is an alternating process of these two methods, on the other hand, some small bubbles in the liquid bridge are also in the moving state by following the fluid due to convection in the liquid bridge. Figure 11 shows the results of bubble removing by both of the two methods interaction, where, Fig. 11a shows the presence of multiple bubbles in the liquid bridge, and Fig. $11 \mathrm{~b}$ and $\mathrm{c}$ show the results of decreased number and decreased size of bubbles. In space experiments, if bubbles are found in the liquid, the temperature control mode for bubble removing will be set to remove bubbles. So, there are three sequences for each sub
Fig. 15 Beat phenomenon (Heating rate $R_{\mathrm{tem}}=0.3 / \mathrm{min}$, $T_{\text {start }}=0 \mathrm{~min}$ )
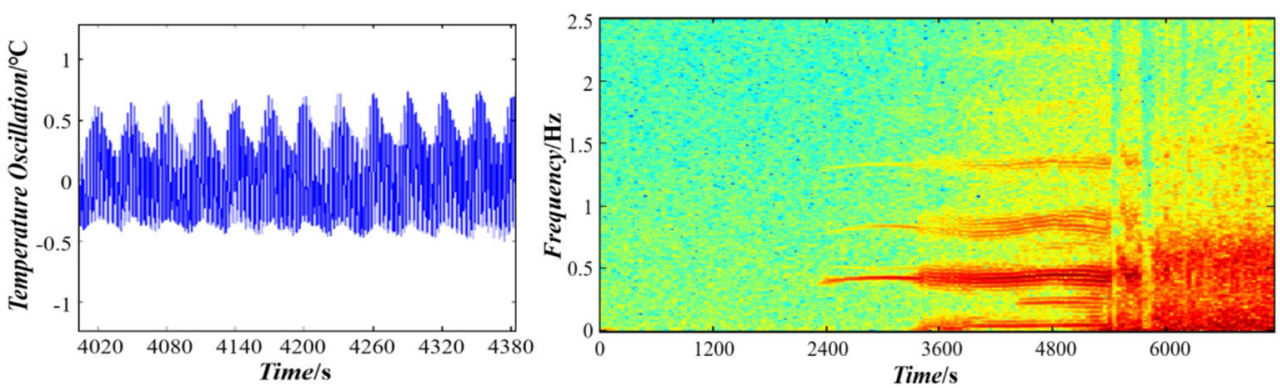
Fig. 16 The period-doubling bifurcation with $1 / 2 \mathrm{f}$ (Heating rate $R_{\text {tem }}=0.3 / \mathrm{min}, T_{\text {start }}=0 \mathrm{~min}$ )
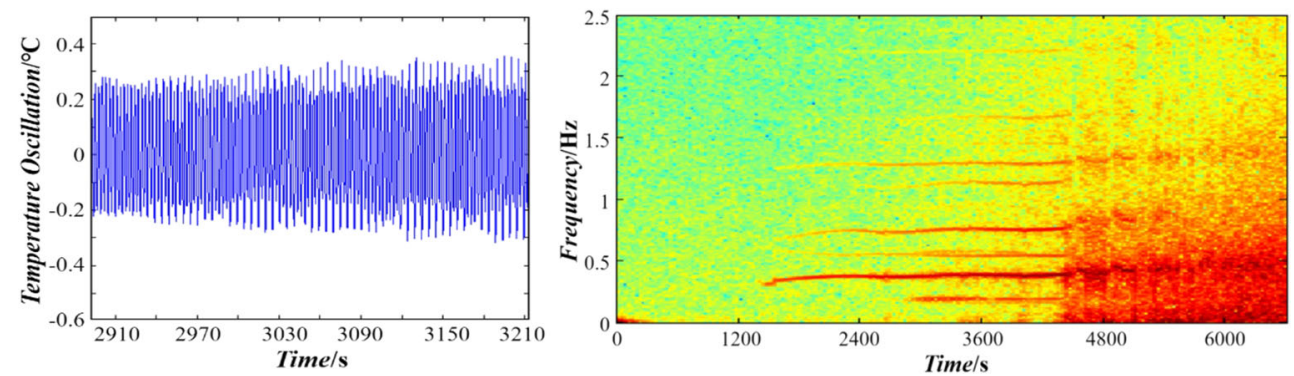

mode of bubble removing to realize temperature differences of 3,6 , and 9 respectively, with a fast changing rate of temperature, $1 / \mathrm{min}$.

\section{Experiment of Temperature Control}

The temperature difference in the liquid bridge is built by heating the upper bridge column with an electrothermal film and cooling the lower bridge column with chilling plates. Temperatures on the upper and lower bridge columns are adjusted through PID control. Multiple temperature control modes are preset in the experiment box, including linear heating, step heating and bubble removing mode. Figure 12 shows the three typical temperature control modes, where, the blue line represents the temperature of the bottom rod, the pink line represents the temperature of the top rod, and the green line indicates the environment temperature.

\section{Ground Matching Experiments and Determination of Fluid Medium in Space}

Which grade of silicone oil will be selected as the fluid medium in space experiments is a very important factor in our study on the oscillation and transition of thermocapillary convection. The decision is finally made through a large amount of ground experiments. For the liquid bridge with a diameter of $20 \mathrm{~mm}$, it is impossible to build the liquid bridge with a large aspect ratio on the ground due to the influence of gravity. After a large number of ground experiments, it is found that the surface tension of $2 \mathrm{cSt}$ and $5 \mathrm{cSt}$ silicone oil is small, and the maximum height of liquid bridge can reach $4 \mathrm{~mm}$ with them, i.e., the aspect ratio is 0.20 ; the surface tension of $10 \mathrm{cSt}$ silicone oil is larger, and the maximum height of liquid bridge can reach $5 \mathrm{~mm}$ with it, i.e., the aspect ratio is 0.25 . Table 1 shows parameters of silicone oil including the viscosity coefficient and the surface tension coefficient.

When the liquid bridge has a temperature difference, $\Delta T$, the convection will be formed driven by thermocapillary force. There is a critical value, $\Delta T_{c}$, and when the temperature difference is increased to $\Delta T=\Delta T_{c}$, the unsteady state starts, and the flow with periodic oscillation appears in the flow field, which expresses as that the fluid temperature signal is in the periodic oscillation. Studying the critical temperature difference $\Delta T_{c}$ at which the oscillation starts has a significant meaning to the study on instability mechanism of thermocapillary convection. On the other hand, obtaining the law of instability in thermocapillary convection is helpful in exploring the new technology of avoiding the appearance of defective structure in crystal growth.

With 2cSt silicone oil, nine sets of ground matching experiments with different aspect ratios and volume ratios have been performed according to the space experimental procedure of liquid filling and bridge establishing. The experimental environment is under $1 \mathrm{~atm}$ and 18 room temperature. The linear heating mode is selected and the heating rate is $0.3 / \mathrm{min}$. The experimental conditions that have been finished with $2 \mathrm{cSt}$ silicone oil are shown in Table 2 as below, and $H$ stands for the height of liquid bridge, $P D$ stands for the pulling distance of the upper bridge column, $V_{O}$ stands for the cylindrical volume of space between the upper and lower columns, $V$ stands for the liquid volume, $I L V$ stands for the injection of liquid volume, $P S$ stands for the piston stroke.

It is found in the experiments that, during the linear heating process, when the temperature difference is greater than a critical value, the phenomenon of temperature oscillation will
Table 3 Matching experiments with $5 \mathrm{cSt}$ silicone oil

\begin{tabular}{lllllllllll}
\hline No. & $\begin{array}{l}H \\
\mathrm{~mm}\end{array}$ & $\begin{array}{l}A r \\
L / D\end{array}$ & $\begin{array}{l}V r \\
V / V_{0}\end{array}$ & $\begin{array}{l}P D \\
\mathrm{~mm}\end{array}$ & $\begin{array}{l}V_{0} \\
\mathrm{~cm}^{3}\end{array}$ & $\begin{array}{l}V \\
\mathrm{~cm}^{3}\end{array}$ & $\begin{array}{l}I L V \\
\mathrm{~mL}\end{array}$ & $\begin{array}{l}P S \\
\mathrm{~mm}\end{array}$ & $\begin{array}{l}\Delta T_{c} \\
{ }^{\circ} \mathrm{C}\end{array}$ & $M a_{c}$ \\
\hline 10 & 3.0 & 0.15 & 0.80 & 0 & 0.9420 & 0.7536 & 0.7536 & 0.6000 & ----- & ----- \\
11 & 3.5 & 0.175 & 0.80 & 0.5 & 1.0990 & 0.8792 & 0.1256 & 0.1000 & 24.49 & 17,652 \\
12 & 4.0 & 0.20 & 0.80 & 0.5 & 1.2560 & 1.0048 & 0.1256 & 0.1000 & 20.28 & 16,704 \\
\hline
\end{tabular}


Table 4 Matching experiments with $10 \mathrm{cSt}$ silicone oil

\begin{tabular}{lllllllllll}
\hline No. & $\begin{array}{l}H \\
\mathrm{~mm}\end{array}$ & $\begin{array}{l}A r \\
L / D\end{array}$ & $\begin{array}{l}V r \\
V / V_{0}\end{array}$ & $\begin{array}{l}P D \\
\mathrm{~mm}\end{array}$ & $\begin{array}{l}V_{0} \\
\mathrm{~cm}^{3}\end{array}$ & $\begin{array}{l}V \\
\mathrm{~cm}^{3}\end{array}$ & $\begin{array}{l}I L V \\
\mathrm{~mL}\end{array}$ & $\begin{array}{l}P S \\
\mathrm{~mm}\end{array}$ & $\begin{array}{l}\Delta T_{c} \\
{ }^{\circ} \mathrm{C}\end{array}$ & $M a_{c}$ \\
\hline 13 & 4.0 & 0.20 & 0.80 & 0 & 1.2560 & 1.0048 & 1.0048 & 0.8000 & ------ & ----- \\
14 & 4.5 & 0.225 & 0.80 & 0.5 & 1.4130 & 1.1304 & 0.1256 & 0.1000 & ----- & ---- \\
15 & 5.0 & 0.25 & 0.80 & 0.5 & 1.5710 & 1.2560 & 0.1256 & 0.1000 & ----- & ----- \\
\hline
\end{tabular}

occur in the liquid bridge with $2 \mathrm{cSt}$ silicone oil; At the same volume ratio, the higher the liquid bridge, the lower the temperature difference for starting oscillation, and the smaller the critical Ma number; at the same aspect ratio, the fatter the liquid bridge, the higher the temperature difference for starting oscillation, and the greater the critical Ma number. Figure 13 shows the experiment results of liquid bridge with aspect ratio being 0.15 and volume ratio being 0.90 . In Fig. 13, the left picture shows temperature signals measured with five thermocouples from the beginning to the end of experiment, and the right picture shows oscillation signals in an interval during the experiment process. Comparing the five thermocouple signals, we can see that there exist obvious phase differences.

In the liquid bridge with 2cSt silicone oil in ground experiments, various bifurcation phenomena are found in thermocapillary convection. When the liquid bridge has an aspect ratio of 0.15 and a volume ratio of 0.80 , tangent bifurcations are found with 1/3f and 2/3f, as shown in Fig. 14, where, the left picture shows original temperature signals, and the right picture shows the time-frequency spectra. When the liquid bridge has an aspect ratio of 0.175 and a volume ratio of 0.80 , the beat phenomenon is observed, as shown in Fig. 15. When the liquid bridge has an aspect ratio of 0.20 and a volume ratio of 0.80 , the period-doubling bifurcation with $1 / 2 \mathrm{f}$ is observed, as shown in Fig. 16.

With $5 \mathrm{cSt}$ silicone oil as the fluid medium, experiments in liquid bridges with three different height, $H=3.0,3.5$, and $4.0 \mathrm{~mm}$, and the volume ratio, $V r=0.80$, have been performed. The experiment results are shown in Table 3 . Because the viscosity of $5 \mathrm{cSt}$ silicone oil is higher than that of 2cSt silicone oil, the phenomenon of temperature oscillation occurs at higher temperature difference. When the height of liquid bridge is $H=3.0 \mathrm{~mm}$, the temperature oscillation doesn't happen; when $H \geq 3.5 \mathrm{~mm}$, the temperature oscillation is found. The temperature oscillation of thermocapillary convection in the liquid bridge with $5 \mathrm{cSt}$ silicone oil is not as orderly as that in the liquid bridge with $2 \mathrm{cSt}$ silicone oil.

With 10cSt silicone oil, in order to observe the temperature oscillation as far as possible, the height of liquid bridge is increased in experiments. Three experiments in liquid bridges with three different height, $H=4.0,4.5$, and $5.0 \mathrm{~mm}$, and the volume ratio, $V r=0.80$, have been performed, as shown in Table 4 . The experiment results show that, in experiments with 10cSt silicone oil as the fluid medium, the oscillation cannot happen at the height of liquid bridge that can be realized on the ground. Because the viscosity of 10cSt silicone oil is higher, the temperature difference required for the appearance of temperature oscillation is higher than the limit of our payload. As a result, in ground experiments, the temperature oscillation is not observed. In addition, bridge breaking happens for the liquid bridge with $H=5 \mathrm{~mm}$ when the heating time is at $75 \mathrm{~min}$.

Through large amount of ground matching experiments, we can see that, in order to carry out study on oscillation and transition processes of thermocapillary convection under microgravity condition in space, not only must the bridge breaking not happen, but also must the oscillation happen, therefore, $5 \mathrm{cSt}$ silicone oil is selected for space experiments is practicable.

\section{Space Experiments}

More than 720 space experiments have been carried out on TG2. The experiment medium is $5 \mathrm{cSt}$ silicone oil, with $\mathrm{Pr}=67$. The range of aspect ratio is $A r=0.20 \sim 1.10$, the range of volume ratio is $V r=0.45 \sim 1.15$, and the heating rate is $0.1 \sim 2.0^{\circ} \mathrm{C} / \mathrm{min}$. The states of liquid bridge with the aspect ratio of 0.8 and the
Fig. 17 CCD images of liquid bridge in space. a The bridge by distant view. b The bridge corner by close shot
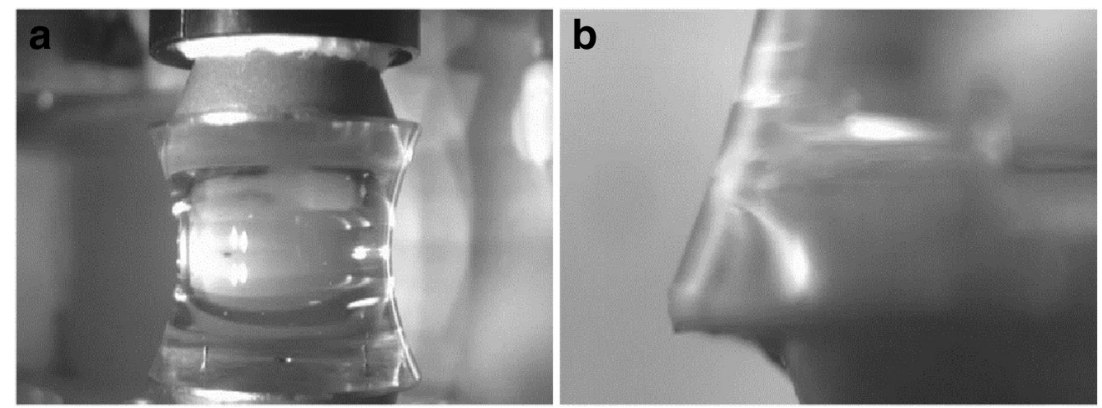
Fig. 18 Temperature and temperature oscillation (Heating rate $\boldsymbol{R}_{\text {tem }}=0.3 / \mathrm{min}, \boldsymbol{T}_{\text {start }}=30 \mathrm{~min}$ )

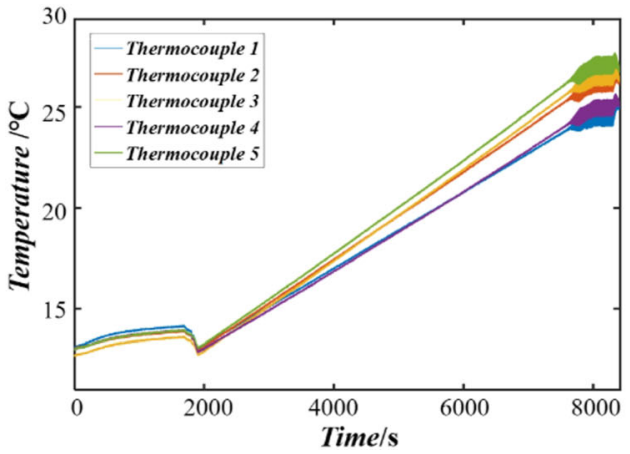

volume ratio of 0.78 obtained from space experiments are shown in Fig. 17, (a) is The liquid bridge by distant view, and (b) is the liquid bridge corner by close shot. It is found in the experiments that, the temperature oscillation occurs when the temperature difference of liquid bridge exceeds a critical value, as shown in Fig. 18, where, the left picture shows temperature signals collected from the beginning to the end of experiment, and the right picture shows signals after the oscillation happens. By analyzing temperature oscillation signals, we can analyze complicated bifurcation and transition paths as well as the aspect ratio effect, volume ratio effect and temperature effect in the transition process. Figure 19a shows the oscillation signal of thermocapillary convection in the liquid bridge with the aspect ratio 0.8 and the volume ratio 0.78 after the time at 9000 second, and the beat phenomenon is observed. Fig. 19b shows the time-frequency analysis results of this experiment, and it is found that quasiperiodic oscillation appears when the time is at 9000 second, and $1 / 2$ period-doubling bifurcation appears when the time is at 10,800 second. The results of space experiments show that there are abundant oscillations, transitions and bifurcations in thermocapillary convection of liquid bridge. A large amount of scientific experiment results about liquid bridges are given in other papers (Kang et al. 2019, 2020).

\section{Conclusions}

This paper introduces the research and development of space experiment payload for studying on thermocapillary convection in liquid bridges with large Pr number on TG-2 space laboratory as well as the experiments. It is confirmed that the objectives of these space experiments are to study the influences of aspect ratio, $A r=L / D$, and volume ratio, $V r=V / V_{0}$, on the critical process of thermocapillary convection in the liquid bridge, and to explore problems of second transition, etc. According to the goals of space experiments, the functions of the payload are analyzed, the components of the payload are required, and the technical and engineering specifications are determined. Designs of wedges, slots and anti-climbing of liquid are applied to the structure of bridge columns to ensure the interface maintenance of liquid bridge during the process of space experiment; the method of bubble removing in liquid is explored, and two modes of bubble removing in space experiments including inter absorption in liquid and escaping from liquid free surface are given; an unique bridge cleaning system is designed, which uses lipophilic medium to absorb silicone oil and ensures the bridge reestablishment after breaking; the aspect ratio and volume ratio of liquid bridge are accurately controlled through liquid filling and bridge pulling by motors; high sensitivity measurement of fluid temperature is realized by high sensitivity thermocouples in space experiments. Multiple functional verification experiments on the payload are carried out to make sure the success of the space experiments. A total of 15 groups of scientific matching experiments with $2 \mathrm{cSt}, 5 \mathrm{cSt}$ and 10cSt silicone oil are carried out on the ground according to space experiment method, and it is found that, in liquid bridges with $2 \mathrm{cSt}$ silicone oil and $5 \mathrm{cSt}$ silicone oil, when the temperature difference exceeds a critical value, buoyant-thermocapillary convection appears temperature oscillation; at the same volume ratio, the
Fig. 19 The period-doubling bifurcation with $1 / 2 \mathrm{f}$ (Heating rate $R_{\text {tem }}=0.3 / \mathrm{min}, T_{\text {start }}=30 \mathrm{~min}$ )
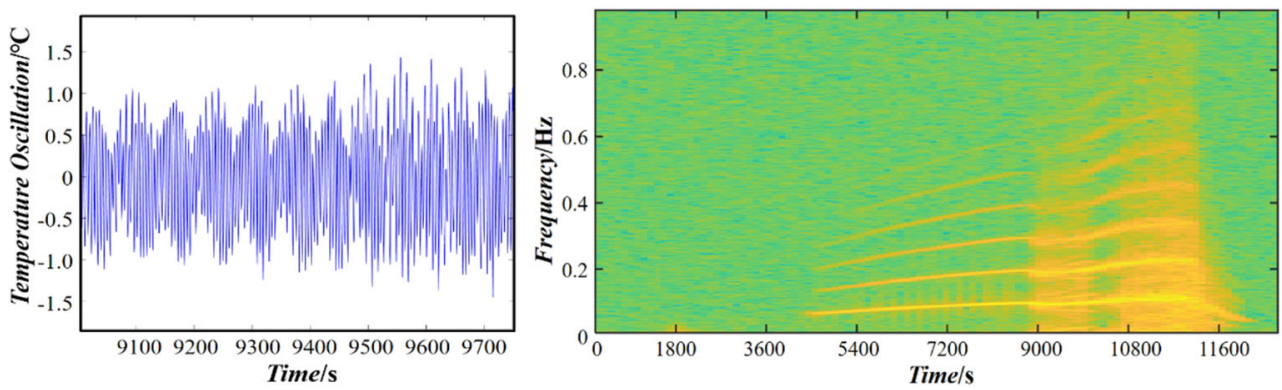
higher the liquid bridge, the lower the temperature difference for starting oscillation, and the smaller the critical Ma number; at the same aspect ratio, the fatter the liquid bridge, the higher the temperature difference for starting oscillation, and the greater the critical Ma number; because the viscosity of $5 \mathrm{cSt}$ silicone oil is a little higher, compared with $2 \mathrm{cSt}$ silicone oil, the critical temperature difference is a little higher with $5 \mathrm{cSt}$ silicone oil; the temperature oscillation signal is not found in experiments with $10 \mathrm{cSt}$ silicone oil, which is because that the viscosity of $10 \mathrm{cSt}$ silicone oil is even higher, and the critical temperature difference is even higher too. According to ground matching experiment results, $5 \mathrm{cSt}$ silicone oil is selected as the experiment fluid medium for space experiments. The liquid bridges states and the temperature oscillation signals obtained from space experiments are given at the end of this paper, and a large amount of scientific analysis results are provided in other papers.

Acknowledgements This project is supported by the China Manned Space Engineering Program.

Open Access This article is licensed under a Creative Commons Attribution 4.0 International License, which permits use, sharing, adaptation, distribution and reproduction in any medium or format, as long as you give appropriate credit to the original author(s) and the source, provide a link to the Creative Commons licence, and indicate if changes were made. The images or other third party material in this article are included in the article's Creative Commons licence, unless indicated otherwise in a credit line to the material. If material is not included in the article's Creative Commons licence and your intended use is not permitted by statutory regulation or exceeds the permitted use, you will need to obtain permission directly from the copyright holder. To view a copy of this licence, visit http://creativecommons.org/licenses/by/4.0/.

\section{References}

Chun, C.H., Wuest, W.: Experiments on the transition from the steady to the oscillatory Marangoni-convection of a floating zone under reduced gravity effect [J]. Acta Astronaut. 6(9), 1073-1082 (1979)

Hu, W.R., Tang, Z.M.: Onset of oscillatory thermocapillary convection[J] 26(4), 85-99 (2013)

Hu, W.R., Shu, J.Z., Zhou, R., et al.: Influence of liquid bridge volume on the onset of oscillation in floating zone convection I. Experiments[J]. J. Cryst. Growth 142(3-4), 379-384 (1994)

Hu, W.R., Tang, Z.M., Li, K.: Thermocapillary convection in floating zones[J]. Adv. Mech. 61(4), 415-422 (2009)

Jiang, H., Duan, L., Kang, Q.: Instabilities of thermocapillary-buoyancy convection in open rectangular liquid layers. Chin. Phys. B 26(11), $114703(2017 a)$

Jiang, H., Duan, L., Kang, Q.: A peculiar bifurcation transition route of thermocapillary convection in rectangular liquid layers. Exp. Thermal Fluid Sci. 88, 8-15 $\Delta \Delta$ (2017b)

Kamotani, Y., Ostrach, S., Pline, A.: Analysis of velocity data taken in surface tension drivenconvection experiment in microgravity. Phys.Fluids 6, 3601-3609 (1994)

Kamotani, Y., Ostrach, S., Pline, A.: A thermocapillary convection experiment in microgravity. J.Heat Trans-T ASME 117, 611-618 (1995)
Kamotani, Y., Ostrach, S., Pline, A.: Some temperature field results from the thermocapillary flow experiment aboard USML-2 spacelab. Adv.Space Res 22, 1189-1195 (1998)

Kamotani, Y., Ostrach, S., Masud, J.: Microgravity experiments and analysis of oscillatory thermocapillary flows in cylindrical containers. J. Fluid Mech. 410, 211-233 (2000)

Kang, Q., Huan, J., Li, D., Chu, Z., Wenrui, H.: The critical condition and oscillation - transition characteristics of thermocapillary convection in the space experiment on SJ-10 satellite. Int. J. Heat Mass Transf. 135, 479-490 (2019)

Kang, Q., Wang, J., Duan, L., Su, Y.Y., He, J.W., Wu, D., Hu, W.R.: The volume ratio effect on flow patterns and transition processes of thermocapillary convection. J. Fluid Mech. 868, 560-583 (2019)

Kang, Q., Wu, D., Duan, L., He, J., Hu, L., Duan, L., Hu, W.: Surface configurations and wave patterns of thermocapillary convection onboard the SJ-10 satellite. Phys. Fluids 31, 044105 (2019)

Kang, Q., Wu, L., Hu, L., Wang, J., Zhang, P., Hu, W.: The effects of geometry and heating rate on thermocapillary convection in the liquid bridge. J. Fluid Mech. 881, 951-982 (2019)

Kang, Q., Wu, D., Duan, L., Zhang, Z., Zhou, B., Wang, J., Han, Z., Hu, L., Hu, W.: Space experimental study on wave modes under instability of thermocapillary convection in liquid bridges on Tiangong2. Phys. Fluids 32, 034107 (2020)

Kawamura, H., Nishino, K., Matsumoto, S., et al.: Report on Microgravity Experiments of Marangoni Convection Aboard International Space Station[J]. J. Heat Transfer 134(3), 031005 (2012)

Nishino, K., Yano, T., Kawamura, H., et al.: Instability of thermocapillary convection in long liquid bridges of high Prandtl number fluids in microgravity[J]. J. Cryst. Growth 420, 57-63 (2015)

Schwabe, D., Scharmann, A., Preisser, F., et al.: Experiments on surface tension driven flow in floating zone melting $[\mathrm{J}]$. J. Cryst. Growth 43(3), 305-312 (1978)

Shevtsova, V., Gaponenko, Y., Kuhlmann, H.C., Lappa, M., Lukasser, M., Matsumoto, S., Mialdun, A., Montanero, J.M., Nishino, K., Ueno, I.: The JEREMI-project on thermocapillary convection in liquid bridges. Part B: Overview on impact of co-axial gas flow. FDMP-Fluid Dyn. Mater. Process. 10(2), 197-240 (2014)

Smith, M.K., Davis, S.H.: Instabilities of dynamic thermocapillary liquid layers. I Convective instabilities[J]. J. Fluid Mech. 132(132), 145$162(1983)$

Wang, J., Wu, D., Duan, L., Kang, Q.: Ground experiment on the instability of buoyant-thermocapillary convection in large-scale liquid bridge with large Prandtl number. Int. J. Heat Mass Transf. 108, 2107-2119 (2017)

Wenrui, H., Renran, Y.: Solutal boundary conditions of marangoni convection in floating zone. Chinese J. Theor. App. Mech. 25(3), 276282 (1993)

Zemei, T., Yan, A., Zhonghua, C., Wenrui, H.: Two bifurcation transition processes in floating half zone convection of larger Prandtl number fluid. Acta Mech. Sin. 18(4), 328-341 (2002)

Zhang, L., Duan, L., Kang, Q.: An experimental research on surface oscillation of buoyant-thermocapillary convection in open cylindrical annuli[J]. Acta Mech. Sinica 5, 681-686 (2014)

Zhu, P., Zhou, B., Duan, L., Kang, Q.: Characteristics of surface oscillation in thermocapillary convection. Exp. Thermal Fluid Sci. 35, 1444-1450 (2011)

Zhu, P., Duan, L., Kang, Q.: Transition to chaos in thermocapillary convection. Int. J. Heat Mass Transf. 57, 457-464 (2013)

Publisher's Note Springer Nature remains neutral with regard to jurisdictional claims in published maps and institutional affiliations. 